\title{
Parinaud's syndrome - a rare presentation of clinically isolated syndrome
}

\author{
Gnanapavan $\mathrm{S}^{1}$, Sillery $\mathrm{E}^{1}$, Acheson $\mathrm{JF}^{2}$ and Toosy $\mathrm{AT}^{3}$ \\ ${ }^{1}$ Department of Neuroimmunology and ${ }^{2}$ Department of Neuro-ophthalmology, National Hospital for \\ Neurology and Neurosurgery, Queen Square, WC1N 3BG. ${ }^{3}$ Department of Brain Repair and \\ Rehabilitation, UCL Institute of Neurology, University College London, WC1N 3BG.
}

\author{
Corresponding author \\ Dr Sharmilee Gnanapavan \\ Department of Neuroimmunology \\ Institute of Neurology \\ Queen Square \\ WC1N 3BG \\ Email: s.gnanapavan@ucl.ac.uk \\ Tel: $+44(0) 7930660210$
}




\begin{abstract}
$\underline{\text { Abstract }}$
We present a 26 year old Pakistani lady with first presentation of a demyelinating syndrome, presenting as Parinaud's syndrome. The video demonstrates a convergent- retraction nystagmus on upgaze, and her MRI brain confirms a corresponding pre-tectal contrast enhancing hyperintense lesion. A review of the literature is presented.
\end{abstract}

\title{
Introduction
}

Parinaud's syndrome or dorsal mid brain syndrome was first described by Henri Parinaud in 1983 [1] with vertical paralysis and/or convergence paralysis and includes a putative case of multiple sclerosis. The case descriptions of the syndrome have since widened to include convergenceretraction nystagmus, pathologic eyelid retraction (Collier's sign) and pseudo-Argyll-Robertson (middilated pupils with light-near dissociation). The differential diagnosis includes pineal gland tumours, MS, acute hydrocephalus, angiomas, infections (e.g. toxoplasmosis), strokes (e.g. thalamic stroke or haemorrhage), and rarely tonic-clonic seizure [2].

\section{Clinical history}

A 26 year old previously well Pakistani lady presented with a one week history of left sided headache and sharp intermittent pains in the occiput. This would progressively worsen through the day and worsen in bright lights. At one stage the headaches worsened and she developed double vision upon looking up and down. There was intermittent nausea but no vomiting. Neurologically she did not complain of visual loss, pain on eye movement or symptoms elsewhere in the body. On examination her visual acuities were $6 / 6+4$ in the right eye and $6 / 9+3$ in the left, with normal colour vision, fundoscopy, pupillary light and accommodation reactions (though sluggish on the left) and visual fields. Examination of ocular motility revealed some upgaze limitation, a mild left over right 3 dioptre vertical concomitant tropia (Figure 1), convergence retraction nystagmus on elevation (video) and convergence insufficiency. 
CT head scan demonstrated an enlarged lesion in the region of the pineal gland which was bright on post-contrast (Figure 2 ) and a subsequent MRI study (Figure 3 ) revealed a T2 hyperintense lesion superiorly on the left side of the mesencephephalon in the periaqueductal region extending into the subthalamic region. An inflammatory panel revealed a mildly elevated ESR 32, but a normal autoantibody panel (ANA, ANCA, dsDNA, RO/LA, SM, RNP, SCL-70 and JO-1, VJKC, NMDAR, glycine, GAD), serum protein electrophoresis, vitamin B12/B1, folate, ACE, thyroid function, HIV, toxoplasma, quantiferon TB Gold, anti-neuronals, HCG and LDH. CSF examination was as follows: clear and colourless, opening pressure 10cm H2O, WCC 6 (lymphocytes), RCC 7, glucose 3.2 (plasma 4.6), lactate 1.31, IgG 50 (normal 10-40), albumin 139 (normal 90-360) and positive oligoclonal bands in the CSF only. Viral PCR (enterovirus, HSV I/II, VZV, CMV, EBV), gram stain, india ink, and AFB were negative. Further imaging in the form of a whole body PET scan was unremarkable.

Her eye symptoms improved over the course of the next few weeks without treatment. A follow up MRI study two months later demonstrated a reduction in the size of the peri-aqueductal lesion with no discernible enhancement. A new non-enhancing lesion was also present in the left cingulate juxtacortical white matter. The combination of findings was most in keeping with an inflammatory demyelinating pathology.

\section{Discussion}

Complex eye movement disorders in the context of MS are not unusual and commonly involve internuclear opthalmoplegias, cerebellar eye movement disorders and pendular nystagmus [3]. Parinaud's on the other hand is an unusual primary manifestation of demyelination with only a handful of cases described $[5,6,7]$. The phenomenon should therefore always be a diagnosis of exclusion in demyelination.

The supranuclear vertical gaze palsy is characteristic in Parinaud's and results from involvement of the rostral interstitial nuclei of the medial longitudinal fasciculus (riMLF), its excitatory efferents 
projecting to the oculomotor nuclei, or their afferent pathways [8]. Attempts at upward gaze produce convergence and globe retraction. Skew deviation may also be present, as demonstrated in our patient, resulting in vertical diplopia.

Treatment is dependent on the underlying aetiology, but in our case, symptoms resolved spontaneously over a period of a few weeks without the need for intravenous steroids.

\section{Figure Legends}

Figure 1: The Hess chart demonstrates a mild left over right tropia responsible for the patient's vertical diplopia.

Figure 2: MRI Brain at presentation: there is a small well circumscribed T2 hyperintense lesion superiorly in the left side of the mesencephalon in the periaqueductal region (COR T2 Flair=Coronal T2 Flair). The lesion does show faint peripheral enhancement (AX T1 FSE + C= Axial fat suppressed T1 post-contrast). The left superior periaqueductal lesion does therefore appear to be a solitary intraparenchymal lesion and an inflammatory lesion would seen more likely than an ischaemic one (SAG T2 FSE=Sagittal T2 fat suppressed).

Figure 3: Follow-up MRI Brain 3 months later: The small left-sided T2- and FLAIR-hyperintense periaqueductal midbrain lesion appears smaller than on the previous. A new small focus of abnormal signal is present within the left cingulate juxtacortical white matter (arrow).

\section{$\underline{\text { Video }}$}

The horizontal saccades are normal, but there is clear convergence-retraction nystagmas on attempted upgaze.

\section{$\underline{\text { References }}$}

[1] Parinaud, H. Paralysie des mouvements associés des yeux. Archives de neurologie, Paris, 1883;5: 145-172. 
[2] Clarke, C., Howard, R., Rosser, M., and Shorvon, S. Queen square textbook of neurology. WileyBlackwell, London, UK.

[3] Barnes, D., McDonald W.I. The ocular manifestations of multiple sclerosis. J Neurol Neurosurg Psychiatry, 1992;55:863-868.

[4] Moreira, I.P., Carneiro, A. and Santos, E. Parinaud Syndrome: A Rare Relapse in Multiple Sclerosis, 2012;36: 133-134

[5] Slyman, J.F., Kline, L.B. Dorsal midbrain syndrome in multiple sclerosis. Neurology 1981;31: 196198.

[6]. Quint, D.J., Cornblath, W.T. and Trobe, J.D. Multiple sclerosis presenting as Parinaud syndrome. AJNR Am J Neuroradiol 1993;14: 1200-1202.

[7] Lee, W.B., Berger, J.R., Lexington, K.Y. and O'Halloran, H.S. Parinaud syndrome heralding MS, Neurology, 2003;60: 322.

[8] Pierrot-Deseilligny, C., Chain, F., Gray, M.,et al. Parinaud's syndrome. Brain, 1982;105:667-696. 
${ }^{*}$ Conflict of Interest/ Role of Funding Source

Conflict of interest: Authors report no conflicts of interest. 

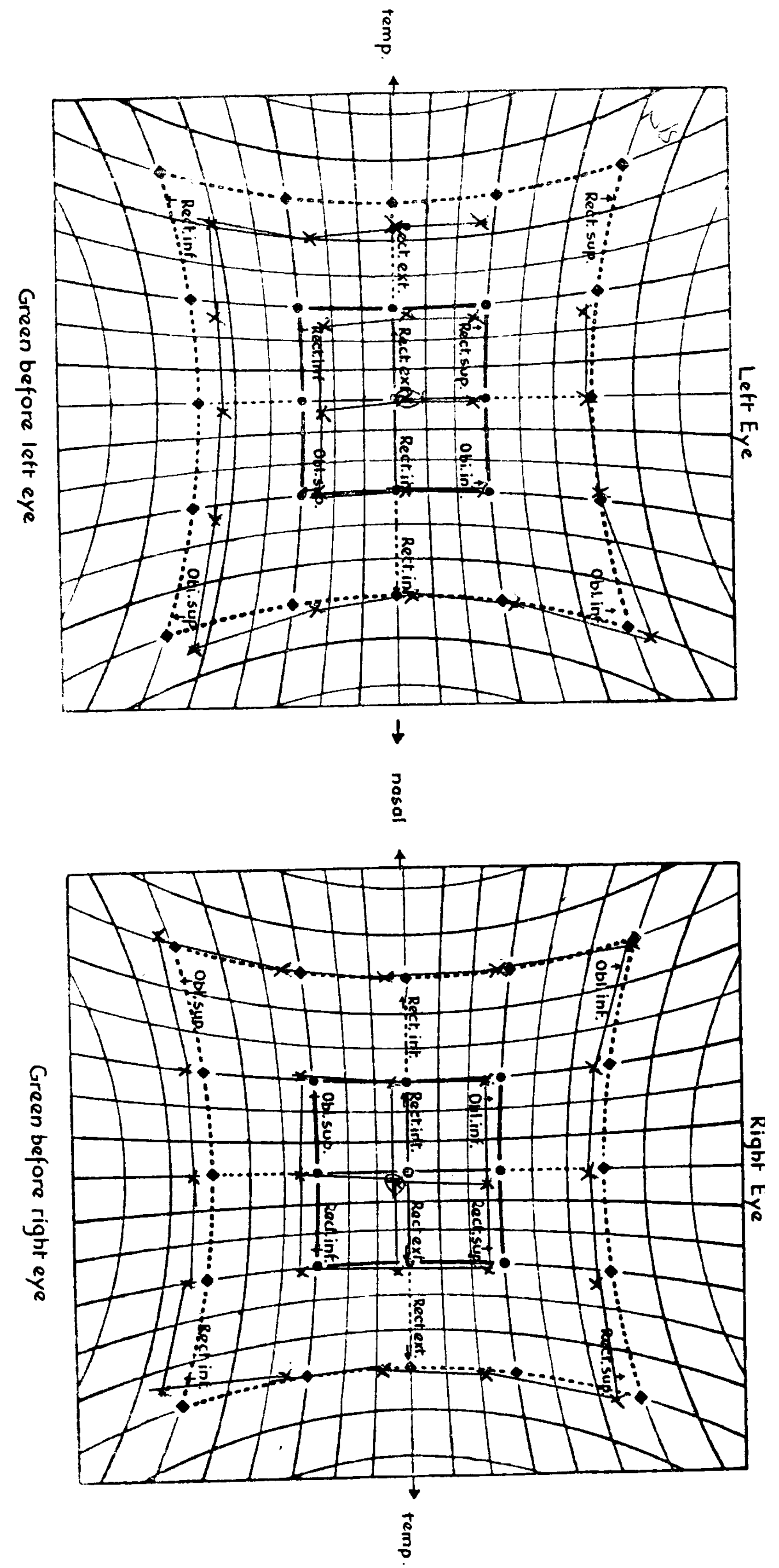
Figure 2

\section{COR T2 Flair}

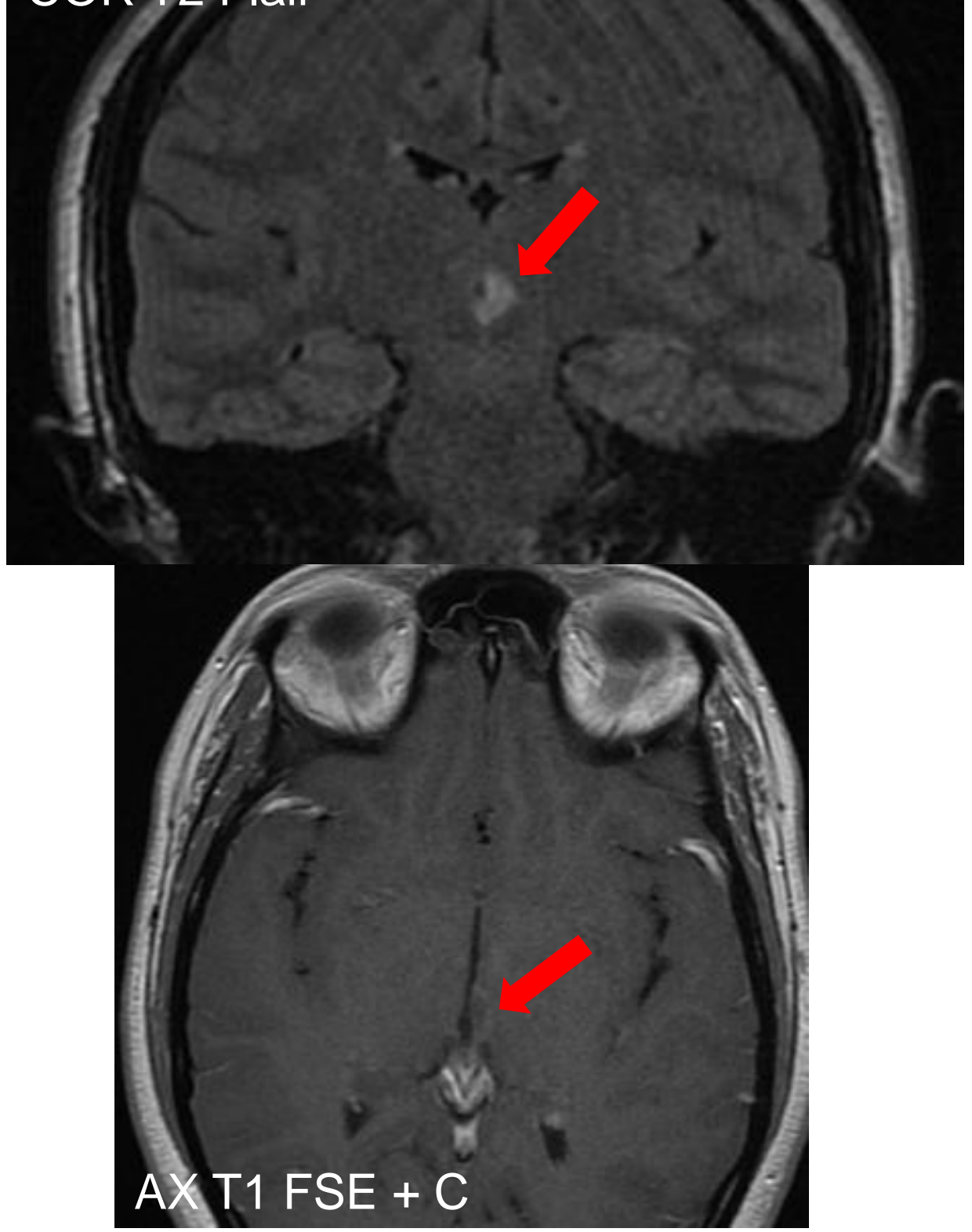

SAG T2 FSE 3

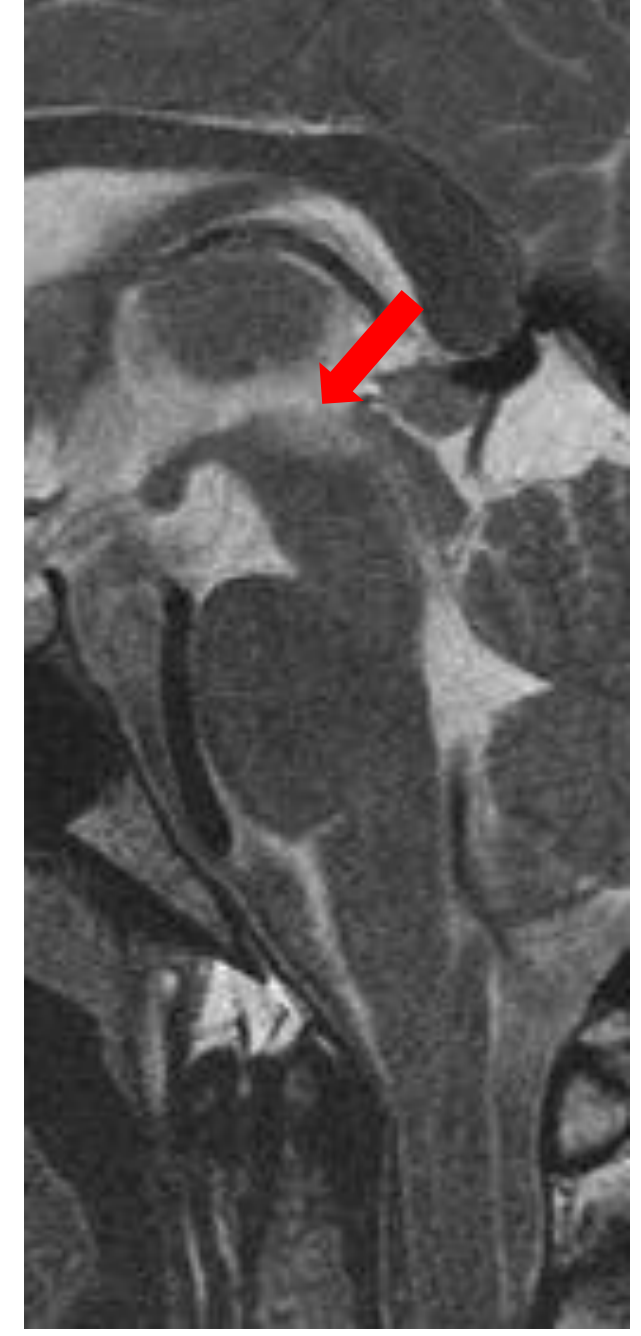


